\title{
Effect of Part Placement Strategy on the Microstructure of Additively Manufactured 17-4PH Stainless Steel Thin-Wall Parts
}

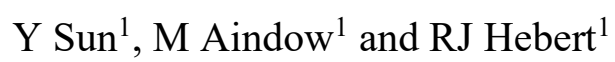 \\ 1. Department of Materials Science and Engineering, Institute of Materials Science, University of \\ Connecticut, Storrs, CT, USA.
}

Laser additive manufacturing (LAM) has attracted considerable interest in the aerospace, automotive and many other industries due to its advantages in producing light weight structural components with complex geometries that are difficult or impossible to manufacture by conventional techniques. In order to reduce weight and manufacturing time, thin-walled structures are some of the most important elements for designing and manufacturing these highly complex AM components. The manufacturing accuracy of AM thin-walled components is known to be influenced significantly by the part placement strategy [1]. On the other hand, the part placement strategy also influences the microstructures of thin-walled components by changing the heat accumulation over the build time, the temperature gradient and the cooling rate of the melt pool. In this work, we investigate the variations in microstructure for AM 17-4PH stainless steel thin walls as a function of the wall thickness and part placement strategy. The correlated texture evolution, phase selection and phase transformations are studied to obtain a comprehensive understanding of the microstructure development of LAM 17-4PH thin walls.

Two sets of thin-wall samples were built to represent the basic part placement strategy in AM component (Fig. 1a). The Set 1 samples were oriented with their edges aligned parallel to the $x$ and $y$ axes of the build plate and the Set 2 sample were oriented with their edges at $45^{\circ}$ to those of the Set 1 samples. Both sets of samples have three $19 \times 19 \mathrm{~mm}$ square vertical walls with thicknesses of 3.2, 1.6 and $0.8 \mathrm{~mm}$ (designated as samples 1, 2 and 3, respectively) built on top of a thick part (Fig. 1b). The samples were produced in a 3DSystems ProX300 SLM machine using the 3DSystems default build theme for 17-4PH powders. The laser scan vectors were parallel to the build plate $\mathrm{x}$ and $\mathrm{y}$ axes and were rotated by $90^{\circ}$ in each successive build layer. In such a build theme, the Set 1 samples have uneven laser scan lengths in each layer, since the laser scans parallel to the long edge in one build layer and parallel to short edge in the next. However, the Set 2 samples have an equal laser scan length in each layer as the scan vectors are always at $45^{\circ}$ to the wall edges. EBSD analysis was performed to study the microstructures of as-built thin-wall samples using a FEI Teneo-LoVac FEG-SEM equipped with an EDAX TEAM ${ }^{\mathrm{TM}}$ EBSD system.

EBSD orientation maps of 17-4PH as-built thin-wall samples are shown in Fig. 2. Set1-1 and Set 2-1 samples both exhibit a non-uniform microstructure composed of coarse columnar grains and fine grains formed along the columnar grain boundaries. As the sample thickness decreases to $1.6 \mathrm{~mm}$, a large volume fraction of fine grains forms in the Set1-2 and Set2-2 samples. With a further decrease in sample thickness to $0.8 \mathrm{~mm}$, both Set1-3 and Set 2-3 samples exhibit a refined microstructure with an average grain size smaller than $10 \mu \mathrm{m}$. Comparing the EBSD orientation maps between Set 1 and Set 2 samples, they exhibit different texture components with most grains in the Set 1 samples aligned with $<100>$ zone axes (grains color coded in red) and most grains in Set 2 samples parallel to $<110>$ zone axes (grains in green). A higher magnification EBSD orientation map of the Set2-3 sample is shown in Figure 3a. The corresponding EBSD phase map in Figure $3 b$ indicates that most of the $\gamma$ phase grains are formed within the larger $\alpha$ phase grains. The orientation relationship for the $\alpha$ and $\gamma$ phase grains in the circled area in Figure 3 is $\{100\}_{\gamma} / /\{110\}_{\alpha}$. This Pitsch orientation relationship indicates that the $\gamma$ phase precipitates in 
the $\alpha$ phase in the as-built 17-4PH thin-wall samples. This $\alpha$ to $\gamma$ phase transformation could have a significant influence on the microstructure development in 17-4PH AM components [2].

\section{References:}

[1] J Kranz, D Herzog and C Emmelmann, Journal of Laser Applications 27(S1) (2014), p. S14001. [2] This work was supported by a research grant from Thermo Fisher Scientific. The microscopy studies in this paper were performed using the facilities in the UConn/ Thermo Fisher Scientific Center for Advanced Microscopy and Materials Analysis (CAMMA).
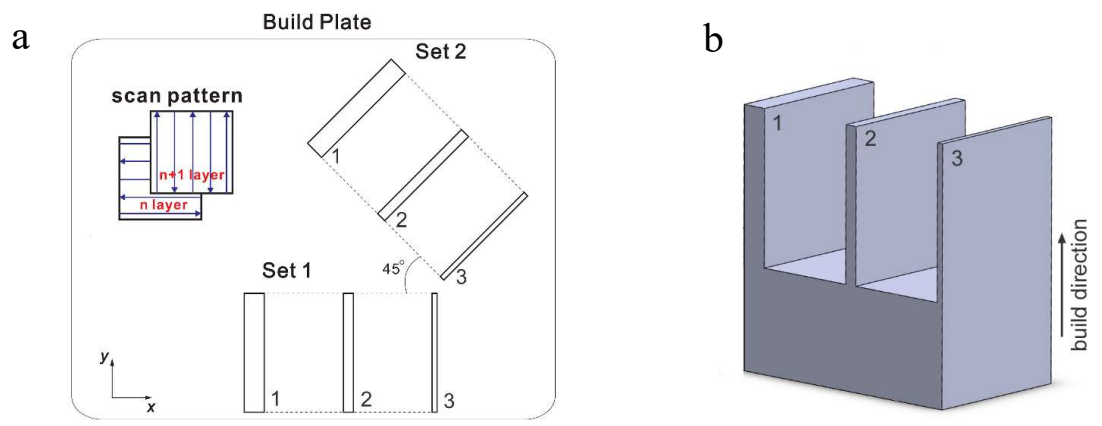

Figure 1. Schematic of (a) part placement strategy and (b) thin-wall sample geometry.
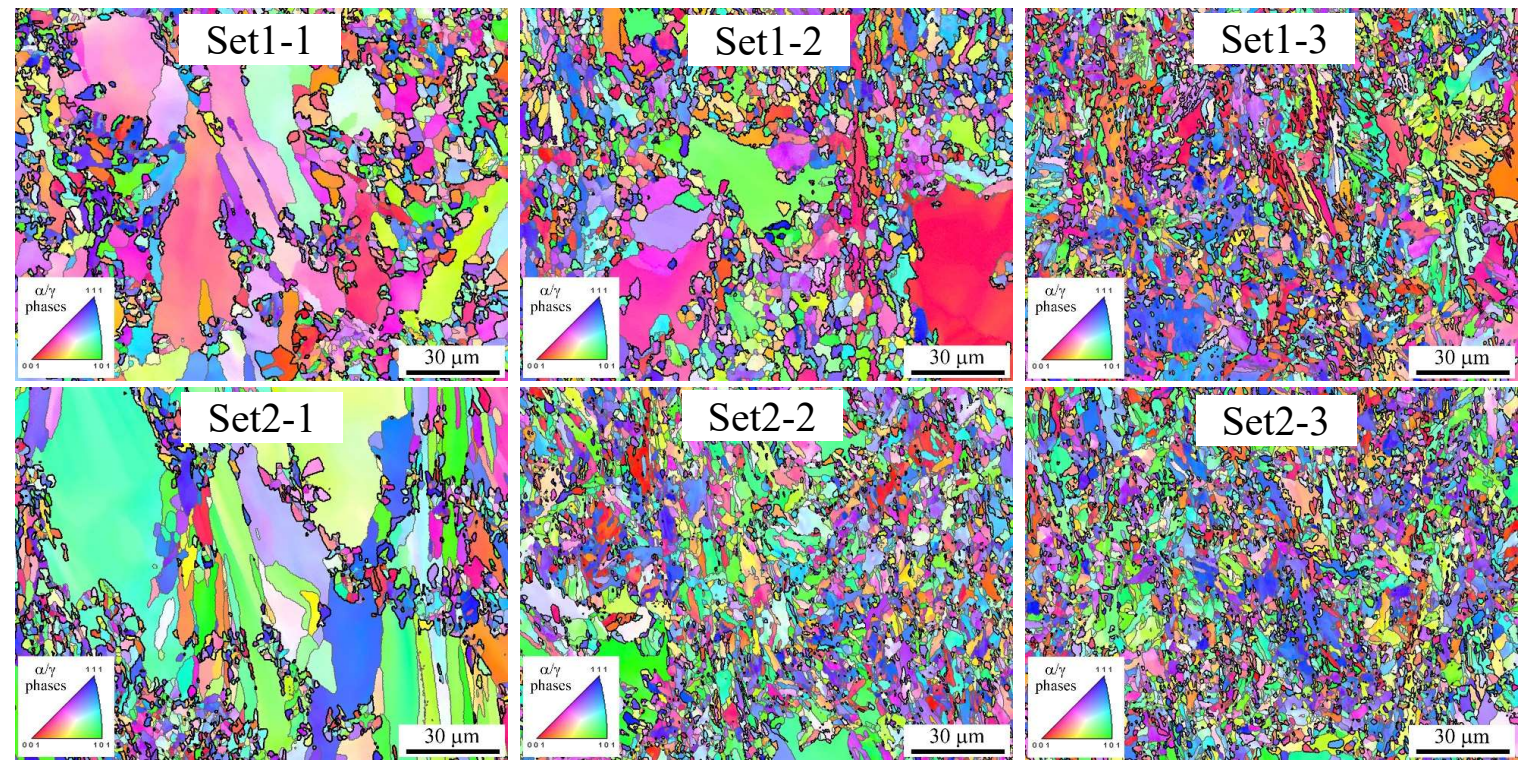

Figure 2. EBSD orientation maps of sections parallel to the walls of the samples (build direction up).
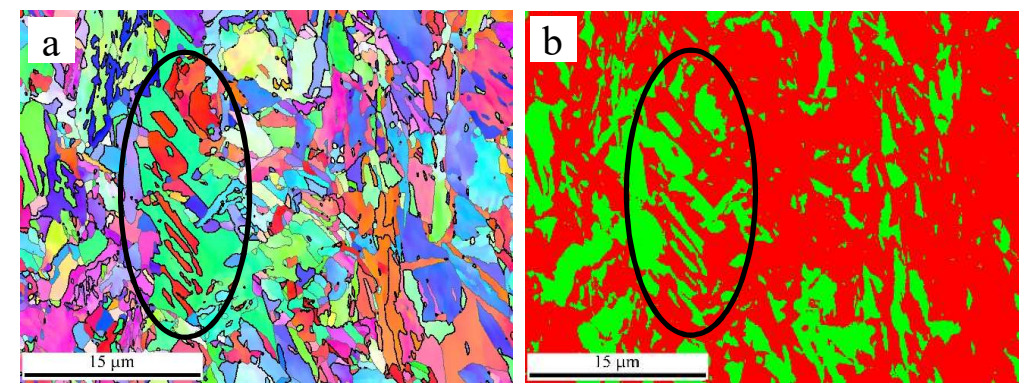

Figure 3. (a) EBSD orientation maps and (b) phase map (Red: $\alpha$ phase, Green: $\gamma$ phase) of Set2-3 sample. 